\title{
La population latino-américaine en Espagne : nouvelles diasporas, nouvelles mobilités
}

Latin-American People in Spain: New Diasporas, New Mobility

La población latino-americana en España: nuevas diásporas, nuevas movilidades

\section{Guillermo Uribe}

\section{OpenEdition}

Journals

\section{Édition électronique}

URL : https://journals.openedition.org/remi/5743

DOI : $10.4000 /$ remi. .5743

ISSN : $1777-5418$

Éditeur

Université de Poitiers

\section{Édition imprimée}

Date de publication : 1 mars 2012

Pagination : 169-178

ISBN : 979-10-90426-03-0

ISSN : 0765-0752

\section{Référence électronique}

Guillermo Uribe, «La population latino-américaine en Espagne : nouvelles diasporas, nouvelles mobilités ", Revue européenne des migrations internationales [En ligne], vol. 28 - $n^{\circ} 1$ | 2012, mis en ligne le 01 mars 2015, consulté le 14 avril 2022. URL : http://journals.openedition.org/remi/5743 ; DOI : https://doi.org/10.4000/remi.5743 


\title{
Note de recherche
}

\author{
La population latino-américaine \\ en Espagne : \\ nouvelles diasporas, nouvelles mobilités
}

\section{Guillermo URIBE ${ }^{1}$}

T es mouvements de population entre l'Espagne et l'Amérique latine s'ins-

Lcrivent dans un contexte historique et culturel particulier. Depuis le XVIe siècle, un flux migratoire soutenu a donné lieu à l'implantation de la culture ibérique en territoire américain. Ainsi, la forte immigration espagnole a été à la base de la création d'une culture hispano-américaine plus ou moins métissée selon les régions. C'est précisément cette proximité culturelle qui a facilité les migrations entre l'Espagne et l'Amérique latine tout au long de l'histoire. Toutefois, la direction des flux a été pendulaire. Pendant près de cinq siècles, ce sont les Espagnols qui ont émigré vers ce continent et le dernier déplacement notoire de population espagnole a eu lieu dans les années 1930-1940². Par la suite, au cours de la deuxième moitié du XXe siècle, le solde migratoire s'est progressivement inversé. En effet, l'Espagne pays d'émigration, a connu un renversement spectaculaire et tout à fait exceptionnel : en moins de deux décennies il est devenu un pays de très forte immigration. C'est ainsi qu'actuellement l'essentiel de cette population immigrée provient de l'Amérique latine et de son versant hispanique en particulier ${ }^{3}$.

Cet article vise à expliciter le contexte de cette immigration et à identifier la constitution récente de nouvelles diasporas ${ }^{4}$.

1 Maître de Conférences en sociologie, Université Pierre Mendès-France de Grenoble, UMR PACTE du CNRS, directeur du Groupe de Recherche et d'Étude sur l'Amérique latine de la MSH ALPES ; guillermo.uribe@upmf-grenoble.fr

2 Plus précisément, au moment de la guerre civile espagnole.

3 Cette immigration n'est pas seulement hispano-américaine, car les Brésiliens sont aussi représentés.

4 Nous avons utilisé les statistiques et les sources officielles disponibles en Espagne, ainsi que celles de certains pays latino-américains et enfin celles des organismes internationaux. Nous avons aussi réalisé un certain nombre d'entretiens et d'observations dans des villes espagnoles. 


\section{L'ESPAGNE NOUVEAU PAYS D'IMMIGRATION}

$\mathrm{Au}$ cours des trente dernières années, l'Espagne a connu un développement économique spectaculaire auquel vient s'ajouter la mondialisation. En effet, un certain nombre de phénomènes concomitants ont contribué à changer radicalement le pays : l'instauration de la démocratie, l'entrée dans le marché commun européen et dans l'Union européenne, puis le passage à l'euro, l'augmentation du niveau de vie et la baisse rapide de la natalité, etc. et l'entrée dans le processus de mondialisation.

De leur côté, les pays latino-américains ont connu au cours de la même période une forte croissance démographique, une augmentation du niveau de vie, une progression significative de la scolarisation, un développement des classes moyennes et une affirmation de la démocratie. Par ailleurs, si la majorité des pays a vécu sous des régimes militaires pendant les années 1970-1980, c'est au cours des trente dernières années que la démocratie s'est affirmée dans le sous-continent ${ }^{5}$. Ces différents éléments ont facilité une nouvelle ouverture vers le reste du monde.

Dans ces conditions, une nouvelle complémentarité s'est créée entre, d'une part l'Espagne, pays ayant connu un développement surprenant accompagné d'une baisse critique de la natalité et, d'autre part, l'Amérique latine qui évoluait dans un autre contexte sociopolitique. À ces éléments vient s'ajouter un nouveau facteur incitatif pour cette émigration latino-américaine traditionnellement orientée vers les États-Unis ${ }^{6}: 1^{\text {'Euro }}$ devient la nouvelle monnaie forte de l'Espagne.

Mais, d'une manière générale, les nouvelles conditions politiques et économiques de l'Espagne ont créé un appel à la main-d'œuvre étrangère. Si en 1981 la population étrangère représentait $0,5 \%$ de la population globale, en 2011 ce pourcentage s'élève à $12,2 \%$. Cet accroissement a été particulièrement important dans les années 2000 : la population étrangère est passée de $2,3 \%$ à $12 \%$ de la population totale ${ }^{7}$. Entre 1997 et 2007, près de 5 millions d'étrangers en provenance de plus de 120 pays se sont installés en Espagne. Ce phénomène a été qualifié notamment par Aja, Arango et Olivier (2008) de « décennie prodigieuse » de l'immigration. C'est ainsi qu'entre 1998 et 2007 l'Espagne occupe le premier rang dans le solde migratoire parmi les pays de l'Union européenne ${ }^{8}$. Toutefois, il convient de noter que les prévisions démographiques actuelles annoncent une décroissance de $1,5 \%$ de la population sur les dix prochaines années ; fait qui serait accompagné par un solde migratoire négatif à partir de $2011^{9}$.

5 Il faut signaler que pendant les années 1960-1980 un bon nombre de ces pays ont vécu des dictatures militaires. Ces régimes autoritaires et les troubles politiques ont déclenché des émigrations forcées sous la forme d'exil politique ; c'est le cas de Cubains, Chiliens, Brésiliens, Argentins et plus récemment de Colombiens et de Vénézuéliens. Les années 1990 ont été marquées par la fin des dictatures avec d'autres formes d'exil et d'émigration.

6 En dehors des migrations transfrontalières.

7 Source : Instituto Nacional de Estadísticas (INE).

8 Source : Eurostat, statistics in focus (2008).

9 Source : Proyección de la población de España a corto plazo 2011-2021, Instituto Nacional de Estadísticas (INE) (2011). 
L'Amérique latine a joué un rôle important dans cet accroissement exceptionnel de l'immigration en Espagne. C'est ainsi qu'en 1980 les immigrants originaires des pays de l'Europe communautaire représentaient $60 \%$ du total des immigrés. Mais, en 2001, ils ne sont que $37 \%$ et dans la même période la population latino-américaine est passée de $17 \%$ à près de $26 \%$ de la population étrangère dans ce pays, ce qui représente la plus forte croissance parmi toutes les origines culturelles ${ }^{10}$. En 2011, les Latino-américains sont, après les Européens, le groupe le plus nombreux, car leur immigration a été la plus stable et la plus constante durant cette période exceptionnelle ${ }^{11}$ (Tableau 1). En outre, cette population témoigne d'un fort enracinement, ce qui contribue actuellement au développement d'un phénomène nouveau dans l'histoire récente de l'Espagne : la diversification de sa population. Cette nouvelle donne devra sans doute marquer l'histoire sociale de l'Espagne.

Tableau 1 : Origine culturelle de la population étrangère résidente en Espagne avant la crise de 2009-2010

\begin{tabular}{|l|c|c|c|c|c|c|c|c|}
\hline $\begin{array}{l}\text { Région } \\
\text { d'origine }\end{array}$ & $\mathbf{1 9 8 1}$ & $\mathbf{\%}$ & $\mathbf{1 9 9 1}$ & $\mathbf{\%}$ & $\mathbf{2 0 0 1}$ & $\mathbf{\%}$ & $\mathbf{2 0 0 8}$ & $\mathbf{\%}$ \\
\hline Europe & 130292 & 65,8 & 180735 & 50,1 & 412522 & 37,2 & 1790267 & 42,9 \\
\hline $\begin{array}{l}\text { Amérique } \\
\text { latine }\end{array}$ & 34762 & 17,6 & 65447 & 18,1 & 283778 & 25,6 & 1230101 & 29,5 \\
\hline Afrique & 5013 & 2,5 & 63054 & 17,5 & 304149 & 27,4 & 865679 & 20,8 \\
\hline $\begin{array}{l}\text { Asie- } \\
\text { Océanie }\end{array}$ & 14149 & 7,1 & 32751 & 9,1 & 92496 & 8,3 & 256252 & 6,1 \\
\hline $\begin{array}{l}\text { Amérique } \\
\text { du Nord }\end{array}$ & 12835 & 6,5 & 17704 & 4,9 & 15021 & 1,4 & 19684 & 0,5 \\
\hline Divers & 891 & 0,5 & 964 & 0,3 & 1095 & 0,1 & 7103 & 0,2 \\
\hline
\end{tabular}

Source : Anuario Estadístico, Ministerio del interior et L. Cachón Rodríguez (2009).

Ainsi, la présence de femmes étrangères en âge de procréer explique que la natalité espagnole se soit fortement développée. Actuellement, $51 \%$ de femmes espagnoles actives n'ont pas d'enfant et $85 \%$ de celles qui en ont déjà décident de ne plus en avoir. Selon María Teresa López (2011) ces raisons sont directement liées au marché de l'emploi. Ainsi, les naissances de mère étrangère sont passées de $4 \%$ en 1998 à $19 \%$ en $2007^{12}$. En 1997, sont nés un peu plus de 15000 enfants de mère étrangère et en 2007 près de 95000 .

10 Cf. Anuario Estadistico de extranjería, Ministerio del interior.

11 Il s'agit de la population en situation régulière, mais la population en situation irrégulière semble importante comme l'a montré en 2005 la régularisation des clandestins : plus de 500000 personnes, dont la moitié était des Latino-américains, ont été concernés. La proportion de clandestins serait d'un illégal pour un légal entre 2003 et 2005 et de un pour trois en 2007 (Izquierdo y León, 2008).

12 Source : Instituto Nacional de Estadísticas (INE). 
Toutefois, les femmes immigrées d'Amérique latine en Espagne ont une fécondité relativement réduite qui n'assure que le remplacement des générations $(2,1)$, mais ce taux fluctue entre 2 et 2,4 selon le pays d'origine. Plus précisément, cette différence est corrélée avec le niveau d'études. Les femmes ayant terminé des études supérieures ont un taux de 1,7 et celles qui ont une scolarisation primaire ou qui n'ont pas fait d'études, ont un taux de $2,7^{13}$. Il convient de préciser que l'immigration latino-américaine est largement une affaire familiale : près de $70 \%$ des foyers sont des couples, $12 \%$ des familles monoparentales et seulement $4 \%$ des personnes seules ${ }^{14}$. Dans ces conditions, cette population affiche une forte représentativité féminine (Tableau 2).

Tableau 2 : Population féminine immigrée en Espagne en 2011 par secteurs géographiques les plus représentés

\begin{tabular}{|l|c|c|c|c|c|}
\hline & Femmes & $\%$ & Hommes & $\%$ & Total \\
\hline Amérique latine & 906080 & 56 & 718990 & 44 & 1625080 \\
\hline Europe & 1135517 & 47 & 1256974 & 53 & 2392491 \\
\hline Afrique & 399942 & 37 & 678957 & 63 & 1078899 \\
\hline Asie & 136936 & 40 & 204950 & 60 & 341886 \\
\hline
\end{tabular}

Source : Instituto Nacional de Estadísticas INE, Cifras provisorias (2011).

Jusqu'en 2008, l'Espagne a été le pays européen qui a connu le plus fort taux d'immigration, grâce à la quantité et à la continuité des flux liés à la demande de maind'œuvre et à la baisse rapide de la natalité espagnole. Alors que les autres pays européens ont développé des politiques restrictives envers l'immigration, l'Espagne a accueilli un bon nombre de ces demandes non satisfaites, ce qui explique la présence d'Asiatiques, d'Africains et de Moyen-orientaux. Pour les Latino-américains, ce pays est devenu à ce moment-là, un lieu privilégié d'émigration en concurrence avec le flux traditionnel vers les États-Unis, ce qui a contribué à élargir la diaspora. La proximité culturelle était un facteur favorable à cette nouvelle immigration, la « latinité », ce fond culturel commun, pouvant se résumer au partage d'une langue, d'une religion et d'un certain type de relations interpersonnelles. Si l'on interroge les immigrants latino-américains en Espagne sur les raisons du choix de l'Espagne, la langue partagée apparaît comme un des arguments principaux.

Dans l'imaginaire de ces nouveaux immigrés, l'émigration vers l'Espagne apparaît comme l'extension de l'émigration vers les États-Unis. Ainsi, ils utilisent pour s'auto-désigner le terme de « latino », nom qu'on leur donne en Amérique du Nord, témoignant par-là de l'intériorisation de l'identité qui leur est attribuée dans cette zone.

13 Cf. Encuesta Nacional de Inmigrantes (2007).

14 Ibid. 


\section{LA CRISE}

À l'instar de ce qui s'est produit dans les autres pays industriels, l'Espagne a souffert de la récession mondiale et de la même façon que l'Irlande, le « miracle » économique espagnol s'est estompé brutalement. Mais cette crise économique qui a touché ce pays depuis 2008 et a abouti à une politique de l'aide au retour des immigrés ne semble pas avoir touché significativement la progression de la population étrangère en Espagne jusqu'en 2011. Ainsi, entre 2007 et janvier 2011, la population étrangère s'est accrue de $27 \%{ }^{15}$, mais le nombre de Latino-américains a diminué notablement entre 2009 et 2010. Est-ce un signe de réactivité à l'évolution du marché de l'emploi ? (Tableau 3).

Tableau 3 : Variation de la population étrangère : 2009-2010

\begin{tabular}{|l|c|}
\hline Équateur & $-10,8$ \\
\hline Argentine & $-9,8$ \\
\hline Colombie & $-7,7$ \\
\hline Pérou & $-5,5$ \\
\hline Brésil & $-3,3$ \\
\hline Cuba & $-1,1$ \\
\hline Europe & $+7,7$ \\
\hline Autres & $-1,5$ \\
\hline Total étrangers & $+2,8$ \\
\hline
\end{tabular}

Source : Secretaría de Estado de Inmigración y Emigración (2011).

En effet selon les entretiens que nous avons conduits avec des migrants latinoaméricains en Catalogne, en Andalousie et à Madrid, nombreux sont ceux qui se rendent en Espagne pour des périodes plus ou moins longues, le temps de réunir des fonds pour réaliser des projets économiques dans leur pays. Il apparaît donc actuellement que l'Espagne n'est plus la destination privilégiée pour ce type de «migration de projet ».

Pour ce qui est des immigrés résidant dans le pays, la situation s'est fortement détériorée depuis 2008 : l'offre de travail a globalement diminué surtout dans les secteurs les plus affectés par la récession telle la construction, gros employeur de main-d'œuvre étrangère. Quant aux immigrés peu qualifiés, ils se sont trouvés en concurrence avec de nouveaux travailleurs espagnols sans emploi. Ainsi, beaucoup de Latino-américains sont rentrés dans leur pays d'origine. À titre d'exemple, fin 2008 en seulement trois mois, plus de 15000 Colombiens avaient pris un billet d'aller simple pour leur pays, selon les statistiques de 2009 de la compagnie d'aviation colombienne Avianca. Mais, plus inattendu, des migrants auraient procédé à des demandes de naturalisation afin de conserver leur

15 Source : Instituto Nacional de Estadísticas (INE). 
droit de résidence. Toutefois, il n'existe pas encore des données chiffrées fiables quant à ce phénomène perçu dans les enquêtes empiriques.

Singulièrement, à l'instar de ce qui s'est produit récemment chez les Espagnols (entre 1950 et 1980), ces immigrés contribuent à diminuer la tension sociale dans leurs pays et sont une importante source de devises grâce aux transferts de fonds destinés à leurs familles.

Malgré la crise actuelle, l'Espagne est le premier pays européen pour les transferts d'argent des travailleurs migrants non européens, ce qui représente bien plus que l'Allemagne, la France et les Pays-Bas réunis ${ }^{16}$. Ainsi l'Espagne est devenue le sixième pays au monde pour l'envoi d'argent réalisé par les immigrés et l'Amérique latine est destinataire de plus de la moitié des sommes envoyées. La main-d'œuvre émigrée est actuellement le deuxième produit d'exportation après le pétrole pour des pays comme le Mexique ou l'Équateur. Pour d'autres, l'émigration est devenue la première source de devises comme c'est le cas du Salvador. Ceci a permis d'affirmer parfois que les travailleurs pauvres, qui ont émigré, apportent plus de devises à leur pays que les investissements des pays industriels ou que l'aide au développement (OCDE, 2007). Mais encore, le caractère de ces envois est bien différencié, car il est possible d'identifier plusieurs types d'immigration à partir des comportements relatifs à l'envoi d'argent. Nous pouvons ainsi observer une immigration à caractère familial qui effectue des transferts de fonds pendant une longue période. C'est surtout le cas des Équatoriens, des Colombiens ou des Péruviens ${ }^{17}$. À côté, il existe une immigration de " rupture », plus individuelle qui transfère peu d'argent. C'est surtout le cas des Argentins (Anta, 2006).

\section{QUI SONT CES IMMIGRÉS ?}

En règle générale, les populations latino-américaines qui émigrent le font parce qu'elles cherchent à améliorer une situation qui ne changerait pas si elles restaient dans leurs pays. L'émigration leur permet de réaliser des économies en vue d'un projet personnel. En d'autres termes, le profil de la population latino-américaine immigrée en Espagne, reflète clairement la situation sociale et économique de leur pays. Ainsi, les pays qui ont plus de $70 \%$ de population urbaine envoient davantage cette population, plus instruite. Tel est le cas pour l'Argentine, la Colombie et le Brésil. Cette situation semble étroitement liée à l'accroissement des classes moyennes dans ces pays. D'autres pays envoient leur reliquat d'exode rural comme l'Équateur ou la Bolivie. Ces derniers migrants, globalement moins instruits, s'emploient davantage dans l'agriculture, la construction ou l'industrie. Pour ce qui est des Argentins, leur profil moyen a évolué récemment : ils sont aujourd'hui plus jeunes, plus nomades et orientés vers les métiers artistiques ou le commerce. En général, l'immigration latino-américaine en Espagne ne correspond pas à une population démunie culturellement. En effet, si la population immigrée en Espagne présente un niveau de scolarisation similaire à celui des Espagnols, les Latino-américains possèdent globalement un niveau de scolarisation plus élevé que celui de la moyenne des Espagnols. Ils ont aussi

16 Le seul pays européen comparable est l'Italie. Source : Eurostat (2011).

17 Dans un bon nombre de cas, ces envois sont fortement liés initialement au remboursement des emprunts pour le financement du déplacement. 
plus d'années d'études que la moyenne de la population de leur pays d'origine. En effet, cette émigration lointaine semble demander plus de capital social, économique et culturel (López Sala, 2005 ; Cea D’Ancona et Valles Martínez, 2009).

Le statut d'occupation du logement peut être un indicateur utile du degré d'enracinement de cette population. Ainsi, $25 \%$ des immigrés latino-américains présents en Espagne en 2007 étaient propriétaires de leur habitation et $55 \%$ étaient locataires ${ }^{18}$, mais avec le prolongement du séjour, la tendance est à devenir propriétaires (Tableau 4).

Tableau 4 : Statut d'occupation du logement des Latino-américains selon leur date d'arrivée

\begin{tabular}{|l|c|c|c|}
\hline Date d'arrivée & avant 1999 & $1999-2002$ & $2003-2007$ \\
\hline Propriétaires & 41 & 27 & 18 \\
\hline Locataires & 50 & 63 & 74 \\
\hline
\end{tabular}

Source : Encuesta nacional de inmigrantes, Ministerio de trabajo y de la inmigración (2007).

La population latino-américaine immigrée en Espagne est loin d'être homogène. Deux facteurs principaux la différencient : le pays d'origine et la catégorie sociale qui se recoupent d'ailleurs en bonne partie. Les contrastes sont flagrants. Les Argentins, les Colombiens et les Péruviens exercent des métiers dans le commerce et les services. Leur profil social est proche de celui des Britanniques vivant en Espagne. Les Équatoriens travaillent dans l'agriculture, le bâtiment et l'industrie, ce qui leur confère un profil plus proche de celui des Marocains ou des Roumains (Graphique 1). L'autre différence est cette fois-ci basée sur la stratification sociale d'origine. En règle générale, les élites ne se mélangent pas avec « la colonie » qui a toutes les caractéristiques de la communauté immigrée. Ce sont les catégories populaires et les petites classes moyennes qui ont tendance à se regrouper et à apparaître comme une minorité visible. On constate ainsi une individuation qui s'accroit au fur et à mesure où l'on monte dans l'échelle sociale.

\section{Graphique 1 : Secteur d'activité des immigrants selon le pays d'origine}

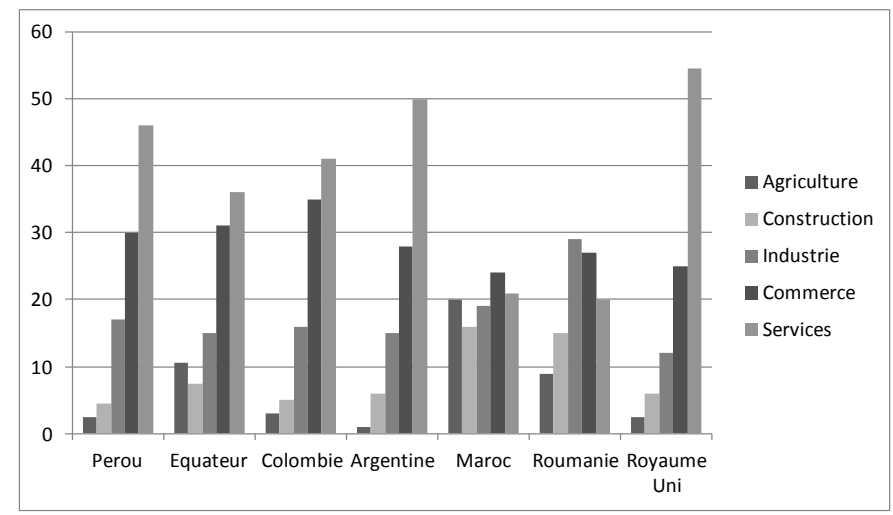

Source : Encuesta nacional de inmigrantes, Ministerio de trabajo y de la inmigración (2007).

18 Encuesta nacional de inmigrantes, Ministerio de trabajo y de la inmigración (2007). 
Les classes populaires occupent la rue et les lieux publics « identitaires », c'està-dire les locaux d'activités culturelles, les épiceries, restaurants et autres commerces de produits de leur pays. Elles donnent aussi une grande importance à la célébration des fêtes nationales. Les magasins d'alimentation et les restaurants sont nombreux. Les classes supérieures, moins nombreuses, ont tendance à se retrouver plutôt dans des lieux privés et ne participent pas aux activités " communautaires ». Mais avec des origines sociales si diversifiées, il est difficile de parler de communautés nationales immigrées. Il ne faut pas oublier que dans certains cas ces immigrés sont des descendants directs d'anciens émigrés espagnols en Amérique latine ${ }^{19}$.

Toutefois, les « latinos $»^{20}$ ont une grande activité culturelle : cercles littéraires, publications, revues, musique, etc. On assiste aussi au retour de la tradition du séjour littéraire et artistique en Espagne qui caractérisait les élites hispano-américaines jusqu'au XIXe siècle. C'est ainsi que des grands écrivains dont plusieurs prix Nobel ont « fait le séjour » madrilène ou barcelonais. Aujourd'hui, arrivent en Espagne pour des séjours volontaires ou en exil politique, écrivains, journalistes, ex-présidents, vedettes des arts, du sport ou de la chanson, ce qui contribue à donner à ce pays l'image d'une terre d'accueil et d'asile.

Il convient de signaler que l'Espagne est un pays de régions culturelles bien différenciées et un pays multilingue. Alors la perception par la population locale de ces immigrées n'est pas exactement la même selon la région dans laquelle elles arrivent. En Catalogne ou dans le Pays basque, la langue espagnole est vécue comme une langue étrangère. Dans ces conditions, un immigré latino-américain, un immigré des îles Canaries ou un Andalou sont souvent perçus de manière similaire. Ainsi la discrimination dont ces immigrés peuvent faire l'objet s'apparente davantage à la domination symbolique de classe qu'à la ségrégation ethnique.

Dans des régions comme l'Andalousie peut s'établir une sorte de condescendance mutuelle entre immigrés et autochtones qui, bien entendu, n'est pas sans comporter des préjugés et des risques de conflits. En règle générale, les rejets publics et collectifs sont rares et lorsqu'ils se produisent, ils semblent plutôt liés à la concurrence d'une maind'œuvre bon marché.

Cette proximité culturelle et cette cohabitation non conflictuelle facilitent des formes d'intégration définitive à la nation espagnole. Pour preuve, le taux élevé des mariages mixtes (Tableau 5).

19 Jusqu'à la fin du XXe siècle, le principe du jus sanguis faisait que les descendants d'Espagnols émigrés avaient la nationalité espagnole. Le flux important d'immigrés latino-américains qui s'est créé pendant ces dernières années a conduit les autorités étatiques à règlementer ce droit. Cela a donné lieu à des milliers de demandes qui ont engorgé les services consulaires dans tous les pays hispano-américains.

20 Cette appellation, née aux États-Unis pour identifier les immigrés latino-américains, a tendance à s'imposer ici, alors qu'en pays latin elle n'aurait pas de sens. 
Tableau 5 : Structure des foyers par nationalités représentatives

\begin{tabular}{|l|c|c|c|c|}
\hline & $\begin{array}{c}\text { Couples } \\
\text { mariés }\end{array}$ & $\begin{array}{c}\text { Femmes } \\
\text { divorcées }\end{array}$ & $\begin{array}{c}\text { Mariés avec } \\
\text { des Espagnols }\end{array}$ & $\begin{array}{c}\text { Personnes } \\
\text { seules }\end{array}$ \\
\hline Argentine & $54 \%$ & $7 \%$ & $34 \%$ & $12 \%$ \\
\hline Colombie & $41 \%$ & $9 \%$ & $35 \%$ & $7 \%$ \\
\hline Équateur & $41 \%$ & $11 \%$ & $14 \%$ & $5 \%$ \\
\hline Pérou & $50 \%$ & $11 \%$ & $24 \%$ & $6 \%$ \\
\hline Maroc & $63 \%$ & $8 \%$ & $9 \%$ & $10 \%$ \\
\hline
\end{tabular}

Source : Encuesta Nacional de Inmigrantes (2007).

On peut se demander si une autre forme de stabilité migratoire n'est pas en train de naître. Elle est proche de ce que certains appellent la " maturité du cycle migratoire » (López Sala, 2005), qui se traduit par une stabilité familiale, une natalité locale, un développement associatif et par l'augmentation des naturalisations.

Quoi qu'il en soit, cette immigration est destinée à durer et aujourd'hui elle est largement composite. Nous pouvons la classer en quatre catégories : les deux plus connues sont l'immigration économique classique qui est la plus fréquente et l'immigration politique assez réduite et très fluctuante. Les deux dernières sont en voie d'émergence : l'immigration de choix de vie, tendance récente chez les classes moyennes et les élites et l'immigration de " mobilité nomade ». Pour cette dernière, l'Espagne devient une tête de pont pour se diriger vers des pays plus intéressants économiquement comme l'Allemagne, l'Angleterre, le Moyen-Orient et l'Asie.

Du coté de la société espagnole, tout semble se passer comme si les Espagnols acceptaient cette immigration soudaine comme faisant partie du «paquet global » de l'ère nouvelle dans laquelle est entrée leur pays depuis trois décennies et que nous avons mentionnée au début de cet article : démocratie, saut en avant économique, entrée dans l'Europe, augmentation du niveau de vie, mondialisation et ... immigration.

Le résultat est une sorte d'élargissement de l'espace ibéro-américain. Peut-être, vivons-nous la préfiguration d'une future aire culturelle transnationale avec des allers retours plus fréquents que ceux que nous a montrés cette longue histoire des mouvements de population? 


\section{Références bibliographiques}

AJA Eliseo, ARANGO Joaquín y OLIVER Josep (2008) La inmigración en la encrucijada. Anuario de la inmigración en España, Barcelona, Fundación CIDOB, 243 p.

ANTA Gregorio (2006) Las remesas de los emigrantes desde España a Iberoamérica, Madrid, Secretaria de Estado de inmigración e inmigración - Remesas.org.

CACHÓN RODRIGUEZ Lorenzo (2009) La «España inmigrante»: marco discriminatorio, mercado de trabajo y políticas de integración, Barcelona, Anthropos, $341 \mathrm{p}$.

CEA D'ANCONA María Ángeles y VALLES MARTINEZ Miguel (2009) Evolución del racismo y la xenofobia en España, Madrid, Ministerio de trabajo e inmigración, 395 p.

DELGADO Lola y LOZANO Daniel (2007) Latinos en España, Madrid, La esfera de los Libros, $284 \mathrm{p}$.

IZQUIERDO ESCRIBANO Antonio y LÉON Sandra (2008) La inmigración hacia dentro: argumentos sobre la necesidad de coordinación de las políticas de inmigración en un Estado multinivel, Política y Sociedad, 45 (1), pp. 11-39.

LÓPEZ María Teresa (2011) Maternidad y trabajo en España y en Europa, Barcelona, Fundación Acción Familiar, 274 p.

LÓPEZ SALA Ana María (2005) Inmigrantes y estados: la respuesta ante la cuestión migratoria, Barcelona, Anthropos, $238 \mathrm{p}$.

OCDE (2007) Les effets économiques de la migration sur le pays d'origine, in La cohérence des politiques au service du développement 2007 : migrations et pays en développement, pp. 55-62.

REHER David-Sven y REQUENA Miguel (Dir.) (2009) Las múltiples caras de la inmigración en España, Madrid, Alianza Editorial, 321 p. 